\title{
Discapacidad intelectual y envejecimiento: un nuevo reto para el siglo XXI
}

\author{
J.C. Millán-Calenti ${ }^{\mathrm{a}}$, L. Meleiro ${ }^{\mathrm{b}}, \mathrm{J}_{\text {. Quintana }}^{\mathrm{c}}$, M.J. López-Rey ${ }^{\mathrm{d}}$, P. Vázquez de la \\ Parra $^{\mathrm{d}}$ y A. López-Sande ${ }^{\mathrm{d}}$ \\ ${ }^{a}$ Catedrático. Escuela Universitaria y Director del Grupo de Investigación en Gerontología (GIG). Departamento de Medicina. \\ Universidad de A Coruña (UDC). España. \\ ${ }^{b}$ Centro de Salud de Coruxo. Vigo. Pontevedra. España. \\ ${ }^{c}$ Departamento de Medicina. Universidad de A Coruña (UDC). \\ ${ }^{d}$ Grupo Investigación Gerontología. Departamento de Medicina. Universidad de A Coruña (UDC). A Coruña. España.
}

\section{RESUMEN}

OBJETIVO: Mediante el presente trabajo se pretende conocer las características de la población con retraso mental que ha supera- do la edad de 45 años.

MÉTODO: Estudio descriptivo de carácter transversal, entre una muestra representativa de 189 personas mayores de 45 años, con certificado de minusvalía que acredite la discapacidad psíquica, seleccionadas aleatoriamente entre las institucionalizadas en los centros de la comunidad autónoma gallega. La herramienta para recoger la información ha sido un cuestionario de elaboración propia, debidamente contrastado.

RESULTADOS: El 60\% de los entrevistados reside con sus familias, con las que mantienen una relación calificada como buena en el $49,7 \%$ de los casos. El 48,7\% presenta un coeficiente intelectual con un retraso moderado, y profundo en el 36,5\%; no se conoce el origen del retraso mental en el $42,85 \%$ de los casos.

CONCLUSIONES: Las necesidades detectadas en la calidad asistencial llevan a concluir la urgencia de actuar sobre los recursos, equipamientos y el grado de especialización de los profesionales implicados, sin obviar a las familias, que constituyen el verdadero soporte asistencial.

\section{Palabras clave}

Envejecimiento, Discapacidad intelectual, Retraso mental, Actividades de la vida diaria, Dependencia.

\section{Intellectual disability and ageing: a new challenge for the twenty-first century}

\begin{abstract}
OBJECTIVE: The aim of this study was to determine the characteristics of adults with intellectual disability who survive for more than 45 years.

METHODS: We performed a cross-sectional, descriptive study of a representative sample of 189 individuals aged more than 45 years with certified intellectual disability. The subjects were randomly selected from individuals attending centres of the Autonomous Community of Galicia in Spain. The instrument used to gather information was a specifically-designed and validated questionnaire.

RESULTS: Sixty percent of subjects lived with their families, with whom $49.7 \%$ reported a good relationship. The degree of intellectual disability was moderate in $49.7 \%$ and severe in $36.5 \%$. Aetiology was unknown in $42.5 \%$.

CONCLUSIONS: Shortfalls detected in the quality of care indicate that urgent action should be taken to improve resources and the degree of specialisation among staff, as well as to aid families, who provide the main support to individuals with mental disability.
\end{abstract}

\section{Key words}

Ageing, Intellectual disability, Mental deficiency, Daily living activities, Dependence. 


\section{INTRODUCCIÓN}

La caída de las tasas de natalidad y mortalidad, junto con el incremento de la esperanza de vida, condicionan el envejecimiento de la población, especialmente en los países desarrollados. En la actualidad, Europa cuenta con más del $20 \%$ de población por encima de 60 años, proporción un poco más alta en España (21,5\%), y sensiblemente más en Galicia $(26 \%)^{1}$.

Por otro lado, la proporción de personas con alguna discapacidad se ha estimado en torno al $10 \%$ de la población total. Se calcula que en el mundo más de 60 millones de personas presentan algún tipo de discapacidad; en España el número asciende a 3,5 millones, y en Galicia, el número total supera los 270.000. Estas cifras experimentarán un significativo aumento en las próximas décadas ${ }^{2}$, debido tanto a los avances tecnológicos como a la mejora de las condiciones higienicosanitarias, que contribuyen al incremento de la esperanza de vida; así, cada vez tendremos más personas con discapacidad psíquica que sobrevivan a sus progenitores.

Con la publicación en 1992 por parte de la Asociación Americana de Retraso Mental (AAMR) ${ }^{3}$ de los conceptos, clasificación y sistemas de apoyo sobre el retraso mental, se ha iniciado una progresión dirigida a mejorar el conocimiento de la conducta adaptativa, la filosofía de los servicios de apoyo y las prácticas en retraso mental. Existe todavía cierta controversia en la aplicación de un término que defina de manera adecuada a la persona con "retraso mental", a pesar de ser muchos los utilizados, según se haga referencia a las concepciones médicas, psicológicas, sociales, educativas... Algunos tienen una connotación muy peyorativa: idiota, imbécil, dismi- nuido, subnormal, retrasado, deficiente...

En la actualidad se tiende a sustituir los términos como "retraso mental" por otras expresiones que conlleven menor carga negativa, como "personas con discapacidad", o "discapacidad en el desarrollo mental", siempre teniendo en cuenta que todas las personas con retraso mental presentan discapacidad intelectual, pero no todas las personas con este tipo de discapacidad están afectadas de retraso mental. Así, el uso de un término u otro dependerá del establecimiento de un diagnóstico previo de retraso mental, o de una desventaja en el desempeño de los roles sociales habituales (minusvalía).

La OMS ${ }^{4}$ utiliza el término "deficiencia" para referirse a una anomalía concreta, como es el caso del síndrome de Down (deficiencia del cromosoma 21, que genera una discapacidad intelectual que se traduce en minusvalía), y a las personas a las que nos referimos en este trabajo les aplica el término de "retraso mental".

La AAMR y el Manual Diagnóstico y Estadístico de los Trastornos Mentales (DSM-IV) ${ }^{5}$ comparten criterios a la hora de definir el retraso mental como "un funcionamiento intelectual general significativamente inferior a la media, que concurre con déficit en la conducta adaptativa, y que se manifiesta durante el período de desarrollo, antes de los dieciocho años", si bien la AAMR matiza que "hace referencia a limitaciones importantes en el desarrollo corriente", y que presenta "limitaciones asociadas en dos o más de las áreas de habilidades adaptativas, como la comunicación, el cuidado personal, la vida en el hogar, las habilidades sociales, la utilización de los recursos comunitarios, el autogobierno, la salud y la seguridad, las habilidades académicas funcionales, el ocio y el trabajo".

En su décima clasificación internacional de las enfermedades, trastornos mentales y trastornos del comportamiento, el CIE- $10^{6}$, aunque no menciona el criterio de edad, sí establece para el diagnóstico definitivo la presencia de un deterioro del rendimiento intelectual que dé lugar a una disminución de la capacidad de adaptación a las exigencias cotidianas del entorno social.

Las personas con discapacidad intelectual presentan características similares a las de la población mayor de 65 años; así, el envejecimiento físico, los ajustes a los cambios biológicos y las necesidades de servicios son similares en las personas ancianas. Las diferencias en esta cohorte se explican, más que por la edad, por el retraso que han padecido durante toda su vida, ya que cada persona vive su propio envejecimiento, y por tanto la variabilidad de éste es la constante en todos los individuos, difiriendo según el origen y el tipo de retraso mental. 
En las personas con discapacidad intelectual se produce una aceleración del proceso fisiológico de envejecimiento ${ }^{7-11}$ que condicionará un incremento de personas envejecidas a edades más tempranas. Este envejecimiento prematuro justifica que se haya establecido ${ }^{12-16}$ su inicio en los 45 años, edad a partir de la cual, a la vez que se produce una repercusión importante sobre la calidad de vida ajustada por discapacidad, se incrementa la tasa de mortalidad.

Si la ancianidad constituye en sí misma un handicap en la sociedad actual, éste se acrecienta si la persona tiene discapacidad intelectual. Y si el hecho de ser anciano se acompaña de la presencia de discapacidad, hechos ambos que favorecen la dependencia, se incrementa el riesgo de institucionalización. Con frecuencia, al llegar la senectud surge la necesidad de una ayuda significativa, en muchos casos importante, para cada uno de los aspectos de la autonomía, y en particular en las actividades de la vida diaria ${ }^{13}$.

El presente trabajo pretende establecer las características del proceso de envejecimiento de personas con retraso mental con respecto a sus relaciones familiares, al uso de los recursos comunitarios, origen del retraso mental, el cociente intelectual y el grado de minusvalía. Por otro lado se pretende conocer el grado de dependencia que, para las actividades de la vida diaria, aparece en relación al proceso de envejecimiento.

\section{MATERIAL Y MÉTODO}

Atendiendo a los intereses y objetivos de esta investigación, nos planteamos como el diseño más adecuado el de carácter transversal y descriptivo. El universo de estudio está constituido por las personas que cumplen los siguientes criterios de inclusión:

1. Ser mayor de 45 años.

2. Estar en posesión del certificado de minusvalía reconocido como disminuido psíquico con discapacidad intelectual.

3. Estar incluido en el concepto de persona con retraso mental.

4. Asistir a uno de los centros de la comunidad autónoma gallega.

A fin de obtener una muestra representativa se empleó la técnica de muestreo probabilístico polietápico. Inicialmente se estratificaron los 116 centros que asisten en Galicia a personas con discapacidad psíquica, atendiendo a criterios territoriales. El primer estrato lo constituyen las cuatro provincias gallegas, y dentro de éste, un nuevo estrato está definido por la condición de rural o urbano. Previamente a la selección de las unidades últimas de muestreo se tiene en cuenta el criterio de régimen de estancia: internado y media pensión. Finalmente se realiza una fijación muestral aleatoria y proporcional, que garantiza la representatividad de la muestra, cuyo tamaño ha quedado fijado en 189 personas.

El universo poblacional está estimado en torno a 450 individuos, de acuerdo con los resultados obtenidos tras recoger los datos por sexo y edad de las personas con discapacidad intelectual institucionalizadas en los centros de la comunidad autónoma gallega, lo que nos per- mite establecer la fiabilidad de los resultados con un margen de error del 5\% y un nivel de confianza del $95,5 \%$.

Dada la inexistencia de un instrumento de medición que cubriera nuestros intereses, para el registro de la información se elabora un cuestionario (anexo 1) adaptado a los objetivos de la investigación, que es previamente sometido al juicio de diferentes expertos en materia de discapacidad. Posteriormente, y a fin de comprobar su correcto funcionamiento, se practica una prueba piloto al $10 \%$ de individuos de la muestra, manteniendo la proporcionalidad de estratos, a fin de que los indicadores de fiabilidad y validez sean idénticos a los de la totalidad de la muestra.

Las variables que se recogen en el mismo hacen referencia a la edad, sexo, tipo de centro al que acude, régimen de convivencia, edad de los padres y relación familiar; recursos económicos, discapacidad mental referida al cociente intelectual (límite, leve, moderado, severo y/o profundo), grado de minusvalía reconocido por las uni- dades de valoración y origen del retraso mental. Igualmente se recogió 
información sobre los recursos que ofrece el tejido comunitario y los servicios prestados a este grupo poblacional. A fin de valorar los aspectos funcionales referidos a las actividades básicas de la vida diaria, se utiliza el índice de Katz por tratarse de una herramienta sencilla, conocida, fiable $(0,73$ y 0,98$)$ y válida $^{17}$.

El trabajo de campo se llevó a cabo entre los meses de abril y junio del 2002.

A fin de completar el estudio se remitió a los centros una ficha específica (anexo 2) referida a datos del mismo, que fue devuelta debidamente cumplimentada.

Una vez finalizado el trabajo de campo, la información fue sistematizada y analizada mediante el paquete estadístico SPSS para Windows versión 11, obteniéndose los resultados que se comentan a continuación.

\section{RESULTADOS}

Con relación a los centros (anexo 2), y atendiendo a la distribución territorial, el 33\% de los centros están ubicados en la provincia de A Coruña; Lugo y Ourense cuentan con un número similar de centros (17 y 18\%, respectivamente), y el restante 32\% se encuentra en Pontevedra. Del total de centros de la comunidad gallega, el 39\% prestan atención residencial y el $61 \%$ ofrecen un servicio de media pensión sin internado, y si atendemos al número de plazas la proporción no varía sustancialmente. A pesar de que la media de plazas en los centros residenciales es muy superior, éstos son mucho menos numerosos que los centros ocupacionales. Actualmente están institucionalizados en Galicia alrededor de 4.000 adultos con distinto grado de discapacidad psíquica, algo más de la mitad en régimen de media pensión.

Sobre la iniciativa o titularidad de los centros cabe decir que únicamente el 3\% es de carácter público; el $88 \%$ pertenece fundamentalmente a asociaciones de padres afectados, y una menor proporción (9\%), a comunidades religiosas. Con la excepción de dos centros -obviamente, de los públicos-, los restantes cuentan con ayudas institucionales a través de los distintos conciertos y convenios que los gobiernos autonómico o local establecen con ellos.

En cuanto al ámbito de competencia, el 43\% de los centros es comarcal, el 30\% autonómico, el 20\% provincial y el $3 \%$ de carácter local.

Con respecto al tipo de plazas ofertadas, el $87 \%$ son de carácter mixto, mientras que el $7 \%$ de los centros únicamente admite hombres y el 6\% únicamente mujeres. La capacidad media ofertada se sitúa alrededor de 40 plazas, en un rango comprendido entre un mínimo de 5 y un máximo de 220.

Sobre los servicios y actividades ofertadas en los centros (tabla 1) cabe destacar que los programas más habituales son los de ocio y tiempo libre, que se ofrecen en la práctica totalidad de los centros. Así mismo ocurre con el servicio de comedor, ya que únicamente el 5\% de los centros no cuenta con este servicio. Los servicios de rehabilitación más comunes son los referidos a estimulación cognitiva, ofertada en el $45 \%$ de los centros, y cuyo objetivo sería mejorar el rendimiento cognitivo y la autonomía personal a través del trabajo sobre la capacidad de comunicación, memoria, atención, percepción sensorial, etc., así como la fisioterapia y la psicomotricidad (50\%).

En lo que respecta a las personas atendidas en los centros de la comunidad (anexo 1), el 57\% son varones y el $42 \%$ mujeres, y la edad media para toda la muestra es de 52,6 años (DS = 7,9): 52,1 años (DS $=6,6)$ para las mujeres, y 53 años (DS $=8,7$ ) para los varones. La distribución por grupos de edad aparece en la tabla 2, en donde el $41,8 \%$ de la muestra tiene menos de 50 años, el 51,85\% entre 50 y 65 años, y el 6,4\% son mayores de 65 años.

Según su convivencia, el 2,6\% viven solos y el 59,8\% con sus familiares, mientras que el 37,6\% están institucionalizados en régimen de internado, por lo que su convivencia se establece en el propio centro. La edad media de los padres es de 80 años, con un intervalo comprendido entre 66 y 98 años; para las madres, la edad media es de 77 años, con un mínimo de 67 y máximo de 92. 
TABLA 1. Oferta de servicios y actividades según los centros

\begin{tabular}{lr}
\hline Servicios y actividades & \% de centros \\
\hline & \\
Transporte & 61,90 \\
Comedor & 95,23 \\
Actividades de ocio y tiempo libre & 95,23 \\
Atención medicosanitaria & 66,66 \\
Atención psicológica & 90,47 \\
Atención social & 85,71 \\
Actividades de formación y habilitación & 90,47 \\
Fisioterapia & 23,80 \\
Apoyo pedagógico & 9,52 \\
Logopedía & 4,76 \\
\end{tabular}

TABLA 2. Distribución de la muestra por grupos de edad

\begin{tabular}{lrr}
\hline Grupo de edad & Frecuencia & $\%$ \\
\hline & & \\
$45-49$ & 79 & 41,80 \\
$50-54$ & 56 & 29,63 \\
$55-59$ & 27 & 14,28 \\
$60-64$ & 15 & 7,94 \\
$65-69$ & 4 & 2,11 \\
$70-74$ & 2 & 1,06 \\
$75-79$ & 1 & 0,53 \\
$80-84$ & 2 & 1,06 \\
85 y más & 3 & 1,59 \\
Total & 189 & 100 \\
& & \\
\hline
\end{tabular}

Respecto a la relación familiar, la definimos como "inexistente" cuando no existe ningún vínculo con el entorno familiar; "mala" cuando la existencia de un discapacitado psíquico origina desestructuración, conflictos o situaciones que dificultan la convivencia de la familia o del discapacitado; "regular" cuando se producen desajustes en la familia, sin dar origen a conflictos que la propia familia no sea capaz de resolver de forma satisfactoria, y por último la relación familiar se define como "buena" cuando la existencia de un discapacitado psíquico no es fuente de conflictos en la familia. De acuerdo con ello, en el $18,2 \%$ no existe relación, en el $12,1 \%$ la relación es mala, en el $22 \%$ de los casos es regular y el $49,7 \%$ refiere que la relación es buena.

En lo que respecta al cociente intelectual, es evaluado mediante la escala de inteligencia de Weschsler revisada (WAIS-R) ${ }^{18}$, obteniéndose que el $14,8 \%$ de la muestra se encuadra dentro del apartado límite $(3,7 \%)$ o leve $(11,1 \%)$, mientras que el $48,7 \%$ presenta un déficit intelectual moderado, y el $36,5 \%$, profundo. Con respecto al grado de minusvalía, en ningún sujeto es inferior al $60 \%$; en el $49,2 \%$ está comprendida entre el 60 y el $75 \%$, y en el $50,8 \%$ es superior al $74 \%$.

Según los resultados obtenidos, en un porcentaje elevado de casos $(42,85 \%)$ no se conoce la causa de la discapacidad intelectual. Entre las causas conocidas, el síndrome de Down $(12,17 \%)$ y las complicaciones del parto $(8,46 \%)$ son las más frecuentes (tabla 3$)$.

En relación a la valoración de las actividades de la vida diaria, de acuerdo con el índice de Katz ${ }^{17}$, los resultados obtenidos pueden observarse en la tabla 4 , en donde el $45 \%$ de la muestra es independiente para todas las actividades evaluadas, por lo que se engloba en el apartado A de Katz, mientras que el 
$10,1 \%$ de la muestra es total- mente dependiente para las actividades de la vida diaria, apartado $\mathrm{G}$ de Katz. Refiriéndonos a cada actividad en concreto, el $52,4 \%$ son dependientes para el aseo, el $38,6 \%$ para vestirse, el 25,5\% para usar el retrete, el 16,9\% para movilizarse, el 20,6\% eran incontinentes y el 13,2\% lo eran para alimentarse.

TABLA 3. Distribución de la muestra de acuerdo con la causa de la discapacidad intelectual

\begin{tabular}{lrr}
\hline Causa del retraso mental & Frecuencia & $\%$ \\
\hline & & 0,53 \\
Autismo & 1 & 8,46 \\
Complicaciones del parto & 16 & 1,59 \\
Epilepsia & 3 & 0,53 \\
Hipotiroidismo & 1 & 6,88 \\
Meningoencefalitis & 13 & 7,94 \\
Parálisis cerebral & 15 & 1,59 \\
Causas prenatales & 3 & 1,06 \\
Psicosis esquizofrénica & 2 & 3,70 \\
Pluripatología & 7 & 42,85 \\
Causa desconocida & 81 & 12,17 \\
Síndrome de Down & 23 & 0,53 \\
Sordomudez & 1 & 1,59 \\
Traumatismo craneoencefálico & 3 & 10,58 \\
No sabe/N contesta & 20 & 100 \\
Total & 189 & \\
\hline
\end{tabular}

TABLA 4. Distribución de la muestra de acuerdo con la clasificación por grupos del índice de Katz

\begin{tabular}{lrr}
\hline Índice de Katz & Frecuencia & \\
\hline & & \\
A & 85 & 45,0 \\
B & 26 & 13,8 \\
C & 24 & 12,7 \\
D & 15 & 7,9 \\
E & 10 & 5,3 \\
F & 5 & 2,6 \\
G & 19 & 10,1 \\
H & 5 & 2,6 \\
Total & 189 & 100 \\
& & \\
\hline
\end{tabular}

\section{DISCUSIÓN}

Es difícil establecer comparaciones con otros trabajos debido a la práctica inexistencia de estudios epidemiológicos publicados sobre las personas con discapacidad intelectual en proceso de envejecimiento. La longevidad de las personas con discapacidad intelectual ha sido estudiada en diversos países, pero no existe un criterio metodológico uniforme ${ }^{19,20}$. Una estimación razonable del número de personas mayores con discapacidad intelectual es de aproximadamente del 0,39\%; es decir, 4 de cada 1.000 ancianos $^{21}$. La prevalencia de la discapacidad intelectual en la población general estadounidense ${ }^{22}$ está comprendida entre el 1 y el $3 \%$. 
En Galicia, el número total de personas con minusvalía psíquica en el año 2000 ascendía a 42.905, según los datos de los Equipos de Valoración y Orientación $(\mathrm{EVO})^{23}$, que recogen únicamente los referidos al número de valoraciones, sin descontar las personas que puedan haber fallecido.

El análisis comparado resulta igualmente difícil por cuanto existe una falta de consenso ${ }^{7,8,10,11}$ sobre la edad a partir de la cual puede considerarse a una persona con discapacidad intelectual como mayor, si bien diferentes autores ${ }^{9}$ se han manifestado a favor de los 45 años como la edad de inicio del proceso de envejecimiento. Sin embargo, el aumento de esperanza de vida y el desarrollo de los apoyos encaminados a mejorar la calidad de vida hacen pensar que esta edad sólo podría aceptarse para los que presentan deficiencias de origen genético. Aquí se ha tomado como referencia esta edad, pero no se ha hecho lo mismo en otras comunidades, por lo que los resultados no son comparables. En cualquier caso, se puede apreciar un importante porcentaje de personas con discapacidad que han iniciado (e iniciarán en breve) su proceso de envejecimiento.

Socialmente se reconoce que una persona es considerada como mayor a partir de los 65 años. Sin embargo, en el caso de las personas con discapacidad intelectual el envejecimiento es más temprano. Nos enfrentamos a una situación en la que cada vez será más frecuente la presencia de personas con un envejecimiento precoz y sin posibilidad de utilizar los servicios sociales por estar éstos diseñados para personas de mayor edad ${ }^{7-11,14-16}$.

La Asociación Americana de Psiquiatría, en su Manual Diagnóstico y Estadístico de las Enfermedades Mentales $^{24}$, clasifica la discapacidad intelectual de acuerdo al cociente intelectual como "leve" cuando el cociente de inteligencia está comprendido entre 50-55 y 70, "moderado" cuando está entre 35-50 y 5055 , "grave" cuando está comprendido entre 20-25 y 35-40, y por último "profundo" cuando se sitúa por debajo de 20-25. Según esta clasificación, los resultados obtenidos en nuestro estudio reflejan un elevado deterioro intelectual, ya que en el $48,7 \%$ de los casos la discapacidad intelectual es moderada, y en el $36,5 \%$, grave y profunda.

La etiología de la discapacidad intelectual es desconocida en la mayor parte de los casos, fenómeno que podemos atribuir al gran desconocimiento que existía hasta hace poco de las causas genéticas ${ }^{25}$ de la deficiencia mental, y a las malas condiciones sanitarias en que nacían los individuos. Coincidimos con otros autores ${ }^{26}$ que no pudieron conocer la etiología en un porcentaje similar, y el grado de desconocimiento es superior en los grupos de mayor edad.

A la hora de conocer las necesidades futuras para las personas con discapacidad intelectual, resulta muy útil saber dónde viven y cuál es la relación que mantienen con su entorno familiar. Al igual que en otras partes de España y del sur de Europa, el régimen de convivencia es mayoritariamente familiar, seguido de la institución residencial. En cuanto a la relación familiar mantenida por las personas con discapacidad intelectual, no se encontró ninguna referencia bibliográfica que haga mención a este dato, de gran importancia a la hora de valorar la repercusión generada sobre el entorno familiar de acuerdo a la carga soportada por el cuidado de la persona con discapacidad intelectual. En los resultados aportados por este estudio, aproximadamente en la mitad de los casos $(49,7 \%)$ se establece una buena relación con la persona con discapacidad intelectual, sin que en la relación influya negativamente la sobrecarga de trabajo por parte de los cuidadores habituales.

El fallecimiento de los padres supone un cambio importante en la vida de las personas con discapacidad intelectual, ya que son ellos los que mayoritariamente asumen los cuidados de estos sujetos, hecho que atribuimos a los cambios sociológicos en la estructura de la familia, que en pocos años ha pasado de ser una familia extensa a una familia nuclear. Si tienen hermanos, en la mayoría de los casos se van independizando, quedando en el domicilio la persona con discapacidad. La edad media de los padres (de casi 80 años) nos sitúa en el elevado envejecimiento de los progenitores, con lo que nos encontramos frente a personas mayores que cuidan de personas mayores, con la consiguiente fragilidad en el sistema de apoyos para la persona mayor con discapacidad intelectual. 
Diversos autores han establecido una correlación importante entre dependencia funcional y sobrecarga del cuidador; de ahí que, habiéndose encontrado un nivel de dependencia importante, podemos presumir que la re- percusión sobre los cuidadores, cuando la institucionalización no es permanente, también lo será. Pero además, la red natural de cuidadores está disminuyendo de acuerdo a diferentes factores sociales; de ahí que, de cara al futuro, sea necesario valorar la demanda de recursos que este grupo poblacional generará sobre el sistema sociosanitario ${ }^{27}$, teniendo en cuenta además que los mayores puedan envejecer en su medio y rodeados de los suyos.

Es necesario incrementar las líneas y los proyectos de investigación en el campo de la discapacidad intelectual, a fin de poder ir homogeneizando los resultados obtenidos hasta el momento y sentar las bases de partida. Es preciso asimismo profundizar en el estudio de la prevalencia de la discapacidad psíquica y de la aparición de enfermedades concomitantes en las personas mayores, que en un futuro no muy lejano van a precisar de recursos y programas que les garanticen un envejecimiento satisfactorio.

Por último, y de acuerdo a las necesidades detectadas, es necesario mejorar la calidad de la atención a las personas con retraso mental, mejorando los recursos, los equipamientos y el nivel formativo de los profesiona- les implicados, en aras de una mayor especialización y multidisciplinariedad.

\section{AGRADECIMIENTOS}

Damos las gracias a todos los centros asistenciales que atienden a personas con discapacidad intelectual que nos han brindado su colaboración para la elaboración de este trabajo, así como a la Sociedad Gallega de Gerontología y Geriatría, con cuya aportación ha contribuido a la realización de este trabajo.

\section{BIBLIOGRAFÍA}

1. Encuesta Nacional sobre Discapacidades, Deficiencias y Estado de Salud. Madrid: Instituto Nacional de Estadística, 1999.

2. Healthy Ageing-Adults with Intellectual Disabilities. Ginebra: OMS, 2000. Summative Report n. ${ }^{\circ}$ WHO/MSD/HPS/MDP/00.3.

3 American Association on Mental Retardation (AAMR). Mental Retardation: definition, classification and systems of supports. 9th ed. Washing- ton: American Association on Mental Retardation, 1992.

4. Organización Mundial de la Salud (OMS). Clasificación Internacional del Funcionamiento de la Discapacidad y la Salud. Ginebra: OMS, 2001.

5. Manual Diagnóstico y Estadístico de los Trastornos Mentales (DSM-IV). Barcelona: Masson, 1995; p. 41-8.

6. Clasificación Internacional de Enfermedades, Trastornos mentales y del Comportamiento, 10. a revisión (CIE-10). Madrid: Meditor, 1998.

7. Wisniewski KE, et al. Envejecimiento normal en adultos con síndrome de Down: un estudio longitudinal. J Intellect Disabil Res 1996;40:208-21.

8. Ribes R, Sanuy J. Indicadores cognitivos del proceso de envejecimiento en las personas con síndrome de Down. Rev Mult Gerontol 2000;10(1):15-9.

9. Ribes R. Nivel de salud y envejecimiento en la discapacidad psíquica. Indicadores en las personas adultas con síndrome de Down. En: Verdugo Alonso MA, Jordán de Uríes Vega, FB, editores. Hacia una nueva concepción de la discapacidad. Salamanca: Amarú, 1999; p. 831-42.

10. Ribes R, Sanuy J. Evaluación del envejecimiento psicológico en las personas con discapacidad psíquica. Rev Mult Gerontol 2000;10(4):242-8.

11. Janicki M, Davison P, Torpe L. Healthy Ageing- Adults with Intellectual Disabilities. Biobehavioural Issues, 2000. Summative Report n. ${ }^{\circ}$ WHO/MSD/HPS/MDP/00.4.

12. Bruckmuller M. Envejecimiento y deficiencia mental. Siglo Cero 1992;140:48-50.

13. Maaskant MA, Van den Akker M, Kessels AGH, Haveman MJ, Van Schorjenstein Lantman-de Valk HMJ, Urlings HFJ. Care dependence and activities of daily living in relation to ageing: results of longitudinal study. J Intellect Disabil Res 1996;40(6):535-43.

14. Moest JC. El envejecimiento precoz de las personas deficientes mentales. Siglo Cero 1987;113:35-9.

15. Janicki MP, Deb S. Ageing: our continuing challenge. Journal of Disability Research 1994;38:229-32.

16. Millán-Calenti JC, Meleiro L, Quintana J. Origen y evolución de la discapacidad intelectual. En: Millán-Calenti JC, editor. Inclusión sociolaboral y envejecimiento en las personas con discapacidad intelectual. Instituto Gallego de Iniciativas Sociales y Sanitarias, 2002; p. 17-35.

17. Katz S, Ford, AB, Moskowitz RW, Jackson BA, Jaffe MW. Studies of ill- ness in the aged: the index of ADL -a standardized measure of biological and psychosocial function. J Am Med Assoc 1963;185:914-19.

18. Wechsler D. A standarized memory scale for clinical use. Journal of Psy- chology 1945;19:87-95.

19. Hogg J, Moss S, Cooke D. Ageing and Mental Handicap. London: Croom Helm, 1988.

20. Eyman RK, Call T, White JF. Life expectancy of persons with Down syndrome. AJMR 1991;95:603-12.

21. Rose T, Janicki MP. Older mentally retarded adults: a forgot population. Ageing Network News 1986;3(5):17-9. 
22. Councilman DL. Caring for adults with mental disabilities. Problems tend to be complex among this growing population. Postgraduate Medicine 1999;106(6):181-90.

23. Consellería de Sanidad y Servicios Sociales. Dirección General de Servicios Sociales. Documento técnico s/n. Santiago, 2000.

24. American Psychiatric Association. Diagnosis and Statistical Manual of Mental Disorder. 4th. Edn. Reviewed (DSM IV). Washington: American Association on Mental Retardation, 1993.

25. Almenara Barrio J, García González-Gordon R, Novalbos Ruiz JP, Mere- llo Martel B, Abellan Hervás MJ, García Ortega C. Evaluación médica y psicosocial de una población adulta con discapacidad intelectual. Rev Esp Salud Pública 1999;73:383-92.

26. Lifshitz H. Aging phenomena among people with mental retardation in community residence in Israel. Education and Training in Mental Retardation and Developmental disabilities 2001;36(1):30-4.

27. Pérez J, Alonso F, Salvador L. Integración y promoción de las personas con retraso mental. Cabra (Córdoba): PROMI; 1997. 


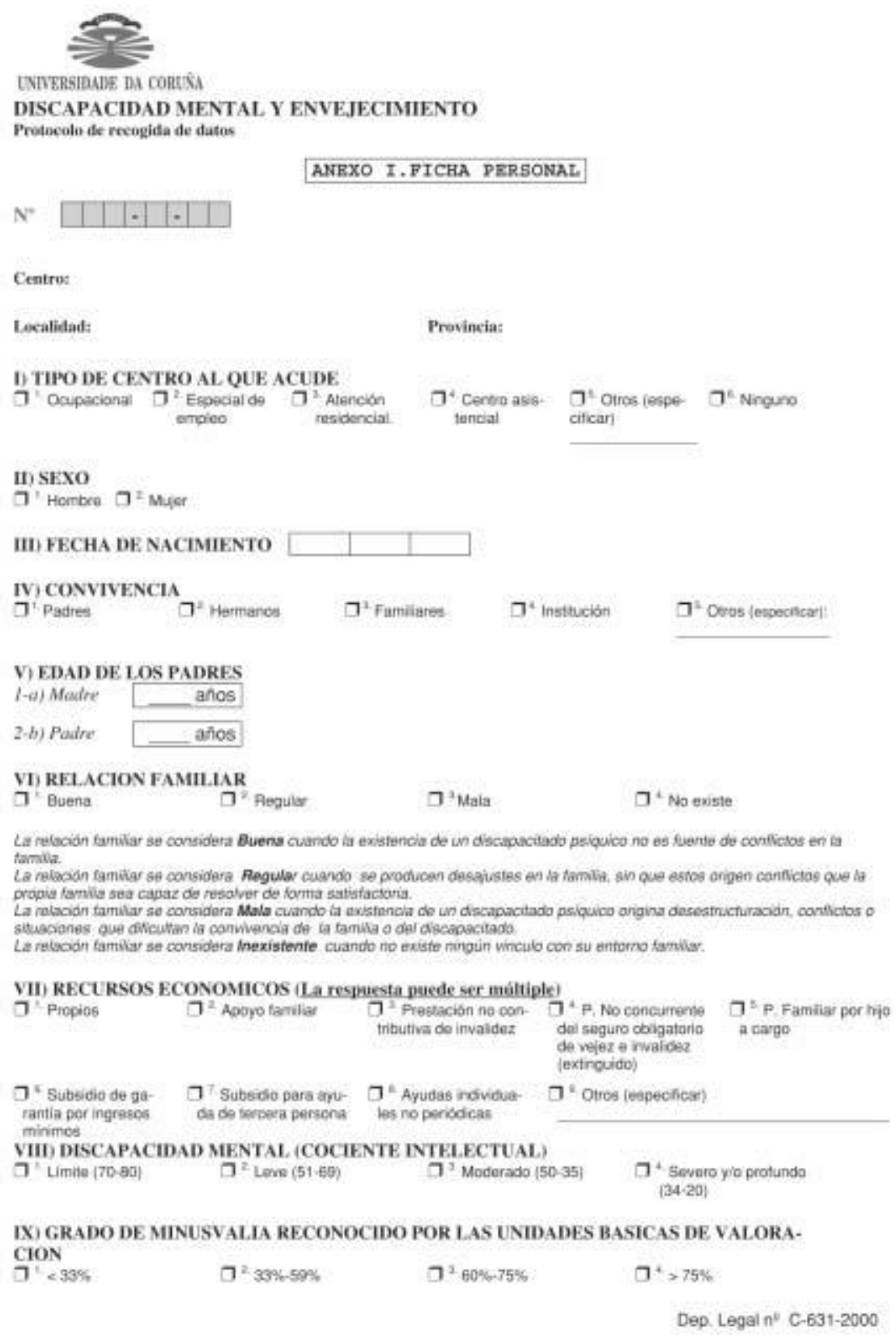


X) ORIGEN DEL RETRASO MENTAL.

$\square^{2}$ Sind. Down $\square^{2}$ Paraliss
cerebral

\section{XI) VALORACION DE LAS ACTIVIDADES DE LA VIDA DIARIA (KATZ)}

1-a) EAÑARSE (com espoujuan, dacha o buñera)

7 'INDEPENDIENTE; Necesta ayuda para tararse una sola parte icomo la espaida o una extremidad incapacitada) o se baha complecamente sin ayude

2-b) VESTIRSE

ᄀ 'INDEPENDIENTE: Coge la ropa de amarias y cajo-

nes, se pone la roos, se pone adornos y abrigos; utliz

nes, se pane la ropa, se pone adarnos y abrigos:

3-c) USAR EL RETRETE

ᄀ '. INDEPENDIENTE: Va al retrete, entra y ralo de at:

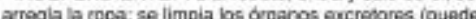
uflizar o no scoportes recairicos!

4-d) MOVILIDAD

$\neg$ 'INDEPENDIENTE: Emira y sale de la cama y se sienta y levanta de la sila independientemente ipuede usar o no scportes mecáricos]

5-e) CONTINENCIA

ᄀ ' INDEPENDIENTE: Contral complona de micoion y dofecacion

$\neg 2$ DEPENDIENTE: Nocesita ayuda para lavarse mats de Gna parte del cuerpo; necesta ayuda para salit o entra en la banera o no se lava soio

$7^{2}$ DEPENDIENTE: No se visie solo o permanece vestdo parcialmente

$\neg^{2}$ DEPENDIENTE: Usa orinal o cuña o precisa ayuda para ir yutilaar al retrate

$\square^{2}$ DEPENDIENTE: Precisa ayuda para utizar la cama yio sila, no realiza uno o más despiazamientoe.

$\square^{2}$ DEPENDIENTE: Incontinencia urinaria o fecal parcial o total; control total o parcial modiante anemas, sondas, o el uso reglado de orinales yo cunas

6-1) ALIMENTACION

7 INDEPENDIENTE: Lueva la conida del plato o su equivalente a la boca (se exclupe de la evaluación e
cortar la carne y la preparación de la comida, como untar mantequila en el pan?

Independiente significa sin saperwision, dirección o asuda personal acrivo

Observaciones:

$7^{2}$. DEPENOIENTE: Precisa ayuda para el aclo de alimentarse no come en absoluto o recibe nutrición pe. renteral

ombre encuestador:

Fecha y Firma: 


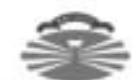

UNVIRSIDADE DA CORLX̃A

DISCAPACIDAD MENTAL Y ENVEJECIMIENTO

Protocalo de recogida de datos

$N^{*}$

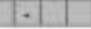

Nembre del Centro:

Directer del Centren

Direccieln:

L.ecalidad:

Códino Postalt

Provineias

Teléfono:

comail:

Fax:

webt

I) TITULAR:

II) TIPO DE CENTRO:

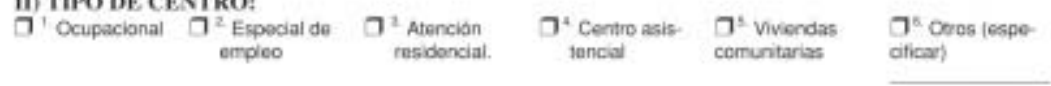

III) CARACTER

(7) Privado $\square^{2}$ Püblico $\square^{2}$ Muto $\square^{2}$ Fundación $\square^{2}$ Otros (Especificar):

IV) AMBITO DE ACTUACION:

$\neg$ Local $\square^{2}$ Comancal $\square^{4}$ Aunonominal $\square^{5}$ Estatal

V) APORTACION ECONOMICA:

$\neg$ Gratuto $\square^{2} \%$ de ingesos del beneficiario $\square^{2} \%$ ingresos de la unidad lamilat $\quad \square^{4}$ Cuota fifa

VI) PLAZAS DEL CENTRO:

ᄀ' Hambres $]^{2}$ Mueres $\square^{2}$ Minto

CAPACIDAD: plaZaE NIPLAZAS OCUPADAS: NF PLAZAS OCUPADAS POA PEASONAS

DE 35 OMAS ANCS CON AETRASO MEN

DE 35 O MAS ANCS CON RETRASO ME

VII) TIPO DE SERVICIOS/ACTIVIDADES

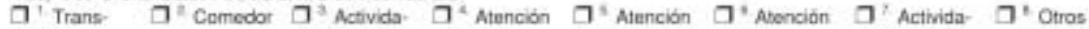

tes de ocisy méder

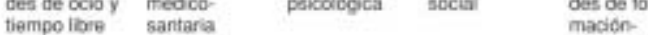

habliatacion

Otservaciones:

Dep. Legal n" C-632-2000 